\title{
Two-dimensional states localized in subsurface layers of $\mathrm{Ge}(111)$
}

\section{$\operatorname{AUTHOR}(\mathrm{S}):$}

Ohtsubo, Yoshiyuki; Yaji, Koichiro; Hatta, Shinichiro; Okuyama, Hiroshi; Aruga, Tetsuya

\section{CITATION:}

Ohtsubo, Yoshiyuki ... [et al]. Two-dimensional states localized in subsurface layers of Ge(111). Physical Review B 2013, 88(24): 245310.

ISSUE DATE:

2013-12

URL:

http://hdl.handle.net/2433/182035

RIGHT:

(C)2013 American Physical Society 


\title{
Two-dimensional states localized in subsurface layers of $\mathrm{Ge}(111)$
}

\author{
Yoshiyuki Ohtsubo, ${ }^{1,2}$ Koichiro Yaji ${ }^{2,3}$ Shinichiro Hatta, ${ }^{1,2}$ Hiroshi Okuyama, ${ }^{1}$ and Tetsuya Aruga ${ }^{1,2, *}$ \\ ${ }^{1}$ Department of Chemistry, Graduate School of Science, Kyoto University, Kyoto 606-8502, Japan \\ ${ }^{2}$ JST CREST, Saitama 332-0012, Japan \\ ${ }^{3}$ Synchrotron Radiation Laboratory, Institute for Solid State Physics (SRL-ISSP), The University of Tokyo, Kashiwa 277-8581, Japan
}

(Received 21 July 2013; revised manuscript received 2 December 2013; published 31 December 2013)

\begin{abstract}
The origin of the two-dimensional surface states localized in subsurface regions of the Ge(111) substrate has been studied by density-functional-theory calculations, which were compared with the experimental results of angle-resolved photoelectron spectroscopy. For the $\mathrm{Bi} / \mathrm{Ge}(111)-(\sqrt{3} \times \sqrt{3}) R 30^{\circ}, \mathrm{Br} / \mathrm{Ge}(111)-(1 \times 1)$, and $\mathrm{Tl} / \mathrm{Ge}(111)-(1 \times 1)$ surfaces, we found that the surface states are classified into three groups. The energy dispersion and the orbital character for each band implies the relationship between the subsurface states and the bulk heavy-hole, light-hole, and spin-orbit split-off bands. These results indicate that the subsurface states originate from the bulk bands that are perturbed due to the truncation of the three-dimensional periodicity at the surface.
\end{abstract}

DOI: 10.1103/PhysRevB.88.245310

PACS number(s): 73.20.At, 71.70.Ej, 79.60.-i

\section{INTRODUCTION}

Surface states are two-dimensional (2D) electronic states localized near the surface region of bulk crystals. Surface states play important roles in determining structural, electronic, magnetic, and chemical properties of surfaces. Two formation mechanisms of surface states can be considered as limiting cases [1]. In a localized-bond picture, surface states can be induced by the atomistic processes such as adsorption of foreign atoms and breaking and alteration of the localized chemical bonds at the very surface layer. The surface states thus formed are usually assumed to have wavefunctions localized in a few topmost layers. On the other hand, in a perturbed-bulkband picture, surface states are derived from bulk Bloch states, which are perturbed by the truncation of the periodic potential at the surface and consequently localized at near-surface region. While surface states in general have both characters with varying relative importance, it is sometimes intuitive to consider these two limiting pictures. For the reconstructed and adatom-covered semiconductor surfaces, experimentally observed surface states have often been discussed in terms of the localized-bond picture at least as a first approximation $[2,3]$, since there have been no clear evidence for surface states of a typical perturbed-bulk-band type. Recently, there is a renewed interest in the surface states originating from bulk bands, which include the spin-split surface states on topological insulators (TI) [4]. Such a type of 2D states are also predicted on a zero-gap semiconductor $\mathrm{HgTe}$ [5].

We have recently found novel surface states around the $\bar{\Gamma}$ point in the surface Brillouin zone (SBZ) on Ge(111) covered with foreign atoms, and showed that these surface states have characteristics typical of the perturbed-bulk-band type [6,7]. The spin polarization of these surface bands was first observed on $\mathrm{Bi} / \mathrm{Ge}(111)-(\sqrt{3} \times \sqrt{3}) R 30^{\circ}[6]$, which exhibited the Rashba-type spin polarization [8] characteristic of 2D surface states. The first-principles calculation showed that these states are localized in subsurface region extending over 10-20 monolayers below the topmost surface. The surface states were found to have negligible contribution of surface

\footnotetext{
*aruga@ kuchem.kyoto-u.ac.jp
}

Bi orbitals, thus the Rashba spin polarization was ascribed exclusively to the spin-orbit interaction (SOI) at the nuclei of $\mathrm{Ge}$ atoms. Similar subsurface states were also observed on $\mathrm{Br} / \mathrm{Ge}(111)-(1 \times 1)$ [7], $\mathrm{Pb} / \mathrm{Ge}(111)-\beta-(\sqrt{3} \times \sqrt{3}) R 30^{\circ}$ $[9,10]$, and $\mathrm{Au} / \mathrm{Ge}(111)-(\sqrt{3} \times \sqrt{3}) R 30^{\circ}[11]$.

In this work, we report on the extensive analysis of the $\bar{\Gamma}$ electronic surface states around the bulk valence-band maximum $(\mathrm{VBM})$ on $\mathrm{Bi} / \mathrm{Ge}(111)-(\sqrt{3} \times \sqrt{3}) R 30^{\circ}, \mathrm{Br} / \mathrm{Ge}(111)$ $(1 \times 1)$, and $\mathrm{Tl} / \mathrm{Ge}(111)-(1 \times 1)$ by using density-functionaltheory (DFT) calculation and angle-resolved photoelectron spectroscopy (ARPES). As shown in Fig. 1, all the surfaces have a common bulk-truncated $\mathrm{Ge}(111)$ substrate structure, and the dangling bonds of the topmost Ge atoms are terminated by the adlayers. The valence of the adatoms is also saturated by the bonding with Ge dangling bonds and among adatoms. Thus the ARPES data near $E_{\mathrm{F}}$ of these surfaces have only limited or negligible contribution from substrate dangling bonds or adsorbate orbitals. Our DFT calculation shows a close relationship between the $\bar{\Gamma}$ subsurface states and the bulk heavy-hole, light-hole, and spin-orbit split-off bands.

\section{EXPERIMENTAL AND COMPUTATIONAL METHODS}

ARPES measurements were performed by using a $\mathrm{He}$ resonance lamp with an energy resolution of $10 \mathrm{meV}$. The clean $\mathrm{Ge}(111)$ surfaces were prepared by repeated cycles of Ar ion sputtering and annealing up to $900 \mathrm{~K}$ until sharp $\mathrm{c}(2 \times 8)$ low-energy electron diffraction (LEED) patterns were observed. The $\mathrm{Bi} / \mathrm{Ge}(111)-(\sqrt{3} \times \sqrt{3}) R 30^{\circ}, \mathrm{Br} / \mathrm{Ge}(111)-(1 \times 1)$, and $\mathrm{Tl} / \mathrm{Ge}(111)-(1 \times 1)$ surfaces were prepared as prescribed elsewhere [7,12-14]. The Tl/Ge(111)-(1×1) surface has two phases with slightly different coverages. In this work, we used the high-coverage phase: The only difference of the two phases in the electronic structure is a shift $(\sim 100 \mathrm{meV})$ of the Fermi level, which does not affect the following discussions. All the LEED patterns and models for the surfaces used in this work are shown in Fig. 1.

DFT calculations were done by using "augmented plane wave + local orbitals" method implemented in the WIEN2K code [15] with SOI taken into account. In order to avoid the underestimation of the bulk band gap, we adopted the 

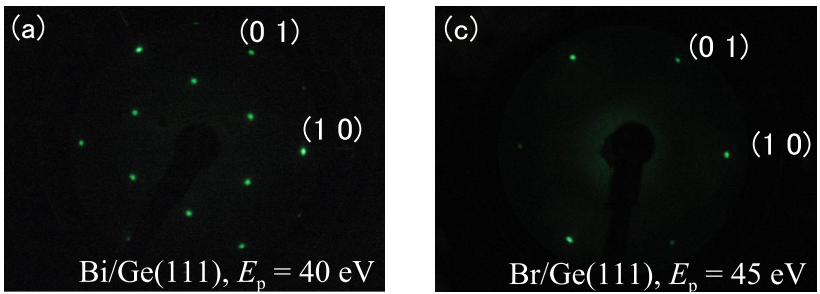

(b)
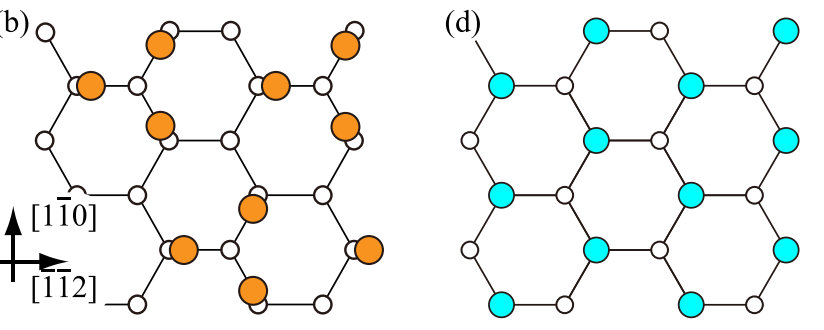

O
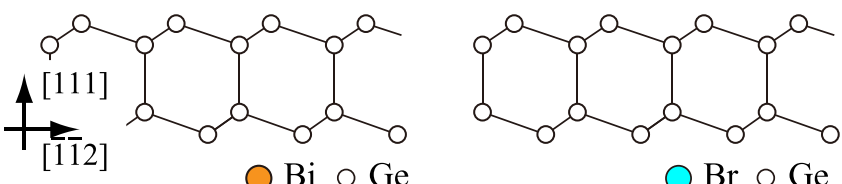

$\bigcirc \mathrm{Br} \circ \mathrm{Ge}$
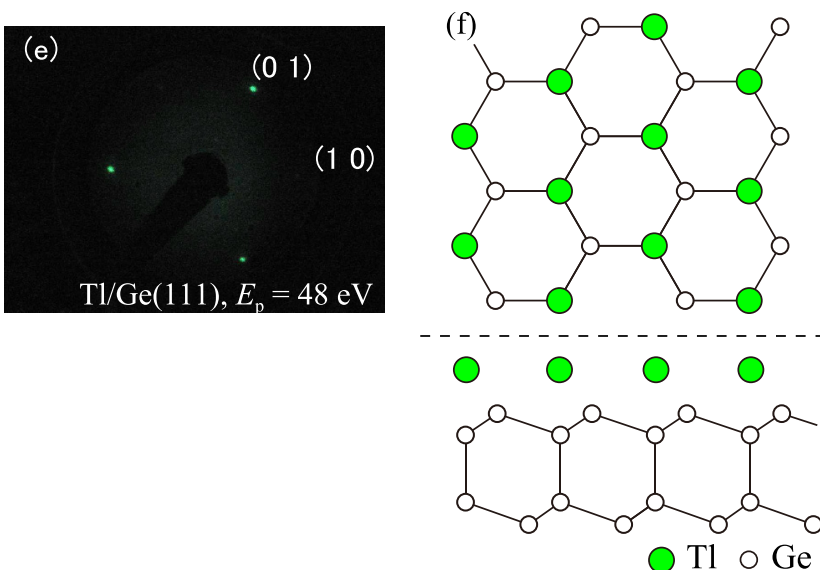

FIG. 1. (Color online) The LEED patterns [(a), (c), (e)] and schematic structures [(b), (d), (f)] of the $\mathrm{Bi} / \mathrm{Ge}(111)-(\sqrt{3} \times \sqrt{3})$ $R 30^{\circ}, \mathrm{Br} / \mathrm{Ge}(111)-(1 \times 1)$, and $\mathrm{Tl} / \mathrm{Ge}(111)-(1 \times 1)$ surfaces. $\mathrm{Bi}$ atoms form trimers, while $\mathrm{Tl}$ and $\mathrm{Br}$ are adsorbed in $(1 \times 1)$ periodicity. All the LEED patterns were captured at room temperature.

modified Becke and Johnson $(\mathrm{mBJ})$ potential together with the exchange-correlation potential constructed by using the local density approximation [16,17]. In this way, we obtained a bulk Ge band gap of $0.75 \mathrm{eV}$, in good agreement with the experimental value of $0.744 \mathrm{eV}$ at $T=0$ [18]. The surface was modeled by repeated slabs with 32 Ge layers each for $\mathrm{Bi} / \mathrm{Ge}(111)$ and $50 \mathrm{Ge}$ layers each for $\mathrm{Br} / \mathrm{Ge}(111)$ and $\mathrm{Tl} / \mathrm{Ge}(111)$. The surface of each slab was covered with a $(\sqrt{3} \times \sqrt{3}) R 30^{\circ}-\mathrm{Bi},(1 \times 1)-\mathrm{Br}$, and $(1 \times 1)$-Tl monolayers, respectively. The other sides of the slabs were terminated with hydrogen for $\mathrm{Bi} / \mathrm{Ge}$ and $\mathrm{Tl} / \mathrm{Ge}$. For $\mathrm{Br} / \mathrm{Ge}(111)$, a symmetric slab with the both sides covered with $\mathrm{Br}$ was used in order to compensate the electric field due to the electronegative $\mathrm{Br}$ adlayer. The atomic structures of the slabs were optimized down to the tenth Ge layer: The optimized structures showed good agreements with those determined previously $[7,19,20]$.

\section{RESULTS}

Figures 2(a), 2(b) and 2(c) shows the band dispersion near $\bar{\Gamma}$ along [1 10$]$ measured by ARPES at room temperature. The second derivative of the photoelectron intensity is plotted in grayscale. The dashed lines indicate the upper edge of the bulk bands calculated with an empirical tight-binding method with parameters adjusted to the experimental bulk band structure [21]. As depicted by solid curves in Fig. 2(d), the valence bands near VBM of Ge are composed of heavy-hole, light-hole, and spin-orbit split-off bands. The light-hole and spin-orbit splitoff bands hybridize with each other to give the energy bands as shown by the dashed curves in Fig. 2(d). While the characters of the latter two bands are interconverted at larger $k$, we label (a)

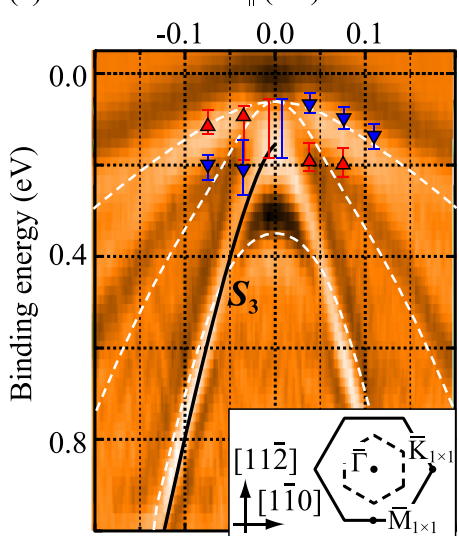

(c)

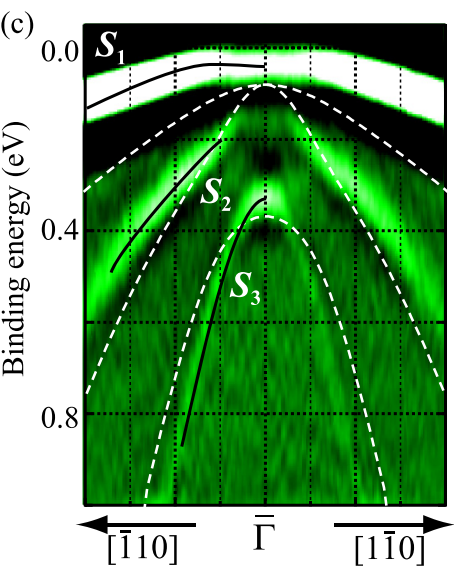

(b) $\quad k_{\|}\left(\AA^{-1}\right)$

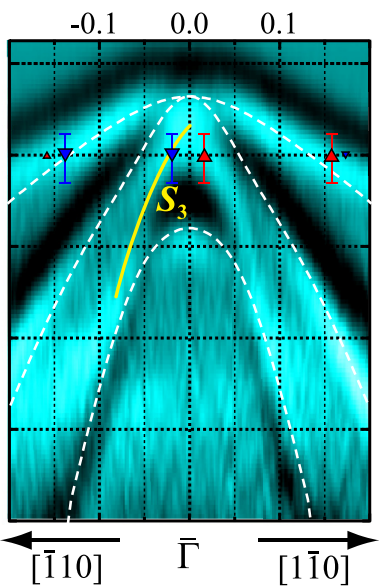

FIG. 2. (Color online) (a)-(c) Second-derivative ARPES images measured with $\mathrm{He}$ I on (a) Bi/Ge, (b) $\mathrm{Br} / \mathrm{Ge}$, and (c) Tl/Ge. Panel (a) was reproduced from Ref. [6]. Solid lines are guides to the eyes for the bands $S_{1}, S_{2}$, and $S_{3}$. Dashed lines correspond to the upper edges of the $\mathrm{HH}, \mathrm{SO}$, and $\mathrm{LH}$ bands, which were calculated based on the tight-binding parameters fitted to the experimental bulk bands. Triangle markers in (a) and (b) represent the spin polarization of each band measured by spin-resolved ARPES as reported in Refs. [6] and [7], respectively. Inset in (a) represents the $(1 \times 1)$ (solid) and $(\sqrt{3} \times \sqrt{3}) R 30^{\circ}$ (dashed) SBZ. (d) Schematic of bulk Ge band structure (see text). At $\Gamma$ the SO band is split by $\Delta_{\text {SO }}=0.29 \mathrm{eV}$ from VBM. 
these bands as $\mathrm{HH}, \mathrm{LH}$, and $\mathrm{SO}$ for convenience. The bulk VBM for $\mathrm{Bi} / \mathrm{Ge}$ and $\mathrm{Br} / \mathrm{Ge}$ are determined to be 50 and $60 \mathrm{meV}$, respectively, below the Fermi level $E_{\mathrm{F}}$ by fitting the calculated bulk bands [7]. On Tl/Ge, an intense surface-state band $S_{1}$ disperses above the bulk bands [19], and the $\mathrm{HH}$ band is less intense than those of $\mathrm{Bi} / \mathrm{Ge}$ and $\mathrm{Br} / \mathrm{Ge}$. Therefore the position of $\mathrm{VBM}$ on $\mathrm{Tl} / \mathrm{Ge}$ cannot be accurately determined by the above method. Instead, we used the energy difference between Ge $3 d_{3 / 2}$ and VBM, which was determined to be $29.87 \mathrm{eV}$ by using $\mathrm{He}$ II for $\mathrm{Bi} / \mathrm{Ge}$ and $\mathrm{Br} / \mathrm{Ge}$. This energy difference corresponds to the energy difference of $29.50 \mathrm{eV}$ between VBM and the centroid of Ge $3 d$, which is consistent with the values reported previously [22,23]. This then was applied to the $\mathrm{Tl} / \mathrm{Ge}$ surface, yielding the VBM position at $70 \mathrm{meV}$ below $E_{\mathrm{F}}$ on $\mathrm{Tl} / \mathrm{Ge}$.

On Bi/Ge, a surface band denoted as $S_{3}$ is clearly found, other than the projected bulk bands. The $S_{3}$ band is positioned at $\sim 100$ meV below VBM at $\bar{\Gamma}$, disperses steeply in the bulk spin-orbit gap, and mostly coincides with SO at $\left|k_{\|}\right|>$ $0.05 \AA^{-1}$. At $\left|k_{\|}\right|<0.05 \AA^{-1}, S_{3}$ disperses into the projected bulk spin-orbit gap. Such a steep dispersion within the bulk spin-orbit gap cannot be ascribed to bulk interband transitions. Similar bands were also observed on $\mathrm{Br} / \mathrm{Ge}$ and $\mathrm{Tl} / \mathrm{Ge}$, as shown in Figs. 2(b) and 2(c), respectively, which are denoted also as $S_{3}$. The $S_{3}$ band on $\mathrm{Tl} / \mathrm{Ge}$ has binding energies larger by $\sim 0.2 \mathrm{eV}$ than those on $\mathrm{Bi} / \mathrm{Ge}$ and $\mathrm{Br} / \mathrm{Ge}$. On Tl/Ge, there are other surface-state bands, labeled $S_{1}$ and $S_{2} . S_{1}$ disperses above $\mathrm{HH}$ and $S_{2}$ between $\mathrm{HH}$ and $\mathrm{LH}$ with an intermediate effective mass between them.

Figure 3 shows ARPES energy distribution curves around $\bar{\Gamma}$ measured on (a) Bi/Ge and (b) Br/Ge. In addition to $S_{3}$ and (a)

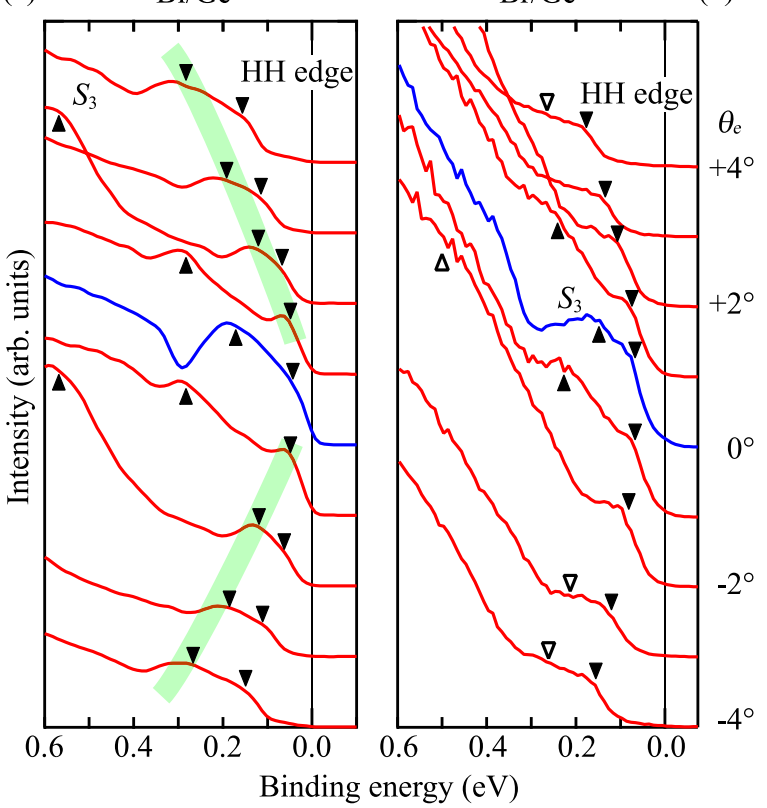

FIG. 3. (Color online) ARPES energy distribution curves measured on (a) $\mathrm{Bi} / \mathrm{Ge}$ and (b) $\mathrm{Br} / \mathrm{Ge}$. Triangle markers indicate peak positions on each spectrum. Empty ones are not observed as an obvious peak but rather weak features. An emission angle $\left(\theta_{e}\right)$ of $3^{\circ}$ corresponds to $k_{\|}=0.1 \AA^{-1}$. the upper edge of $\mathrm{HH}$, there are another components dispersing steeper than $\mathrm{HH}$, as indicated in Fig. 3(a). They touch $\mathrm{HH}$ at $\left|\theta_{e}\right|=1^{\circ}\left(\left|k_{\|}\right|=0.04 \AA^{-1}\right)$ and overlap the spin-polarized states with Rashba spin splitting observed by spin-resolved ARPES [6] [triangle markers in Fig. 2(a)]. This component exhibits energy dispersion similar to $S_{2}$ on Tl/Ge. Binding energies of $S_{2}$ on $\mathrm{Tl} / \mathrm{Ge}$ are larger by $\sim 0.1 \mathrm{eV}$ than those anticipated for $\mathrm{Bi} / \mathrm{Ge}$. While there are no obvious peaks corresponding to $S_{2}$ on $\mathrm{Br} / \mathrm{Ge}$ [see Fig. 3(b)], the HH edges at $\theta_{e}= \pm 4^{\circ}$ show longer skirts than those at $\theta_{e}= \pm 2^{\circ}$ on the high binding energy side. It enables us to assume weak components in addition to the HH upper edge, as indicated by empty triangles in Fig. 3(b). The previous spin-resolved ARPES measurement on $\mathrm{Br} / \mathrm{Ge}$ [7] [see triangle markers at $k_{\|}= \pm 0.14 \AA^{-1}$ in Fig. 2(b)] also suggests the existence of a surface band dispersing around the upper edge of $\mathrm{HH}$.

In order to further establish and characterize these surface states, we have performed first-principles calculations. Figures 4(a)-4(f) show the calculated states along [110] on $\mathrm{Bi} / \mathrm{Ge}, \mathrm{Br} / \mathrm{Ge}$, and $\mathrm{Tl} / \mathrm{Ge}$ slabs, respectively. The radii of the circles $R_{k_{\|}, E}^{ \pm}$are defined by

$$
R_{k_{\|}, E}^{ \pm} \propto\left|\sum_{i=0}^{n}\left(\left|\left\langle\phi^{i}, \uparrow \mid \Psi_{k_{\|}, E}\right\rangle\right|^{2} \pm\left|\left\langle\phi^{i}, \downarrow \mid \Psi_{k_{\|}, E}\right\rangle\right|^{2}\right)\right|
$$

where $\left|\phi^{i}, \uparrow\right\rangle\left(\left|\phi^{i}, \downarrow\right\rangle\right)$ represents the atomic orbital in the $i$ th layer (the zeroth layer corresponds to the adlayers) with spin polarization toward [112] $([\overline{1} \overline{1} 2])$, and $\left|\Psi_{k_{\|}, E}\right\rangle$ is the eigenfunction of the calculated state at $\left(k_{\|}, E\right)$. We summed the atomic orbitals up to $n=6$ for $\mathrm{Bi} / \mathrm{Ge}$ and $\mathrm{Br} / \mathrm{Ge}$ and $n=10$ for $\mathrm{Tl} / \mathrm{Ge}$, since the penetration depth for the $\mathrm{Tl} / \mathrm{Ge}$ is longer than those for the other surfaces as shown later. $R_{k_{\|}, E}^{+}$corresponds to the total atomic contribution of the surface and subsurface layers, and $R_{k_{\|}, E}^{-}$corresponds to the net spin polarization. Thus, the large circles in the panels $(\mathrm{a}-\mathrm{c})$ represent the states which are localized in the surface and subsurface layers, and those in the panels $(\mathrm{d}-\mathrm{f})$ represent their net spin polarization toward

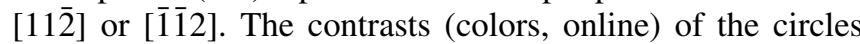
represent the direction of the spin polarization. On the left side, the bulk bands projected onto the (111) surface are also shown (thin lines). Solid lines in the right side represent the upper edges of the bulk bands. The positions of VBM are set to the experimental values and the energies of the slab states are shifted according to them.

For $\mathrm{Bi} / \mathrm{Ge}(111)$ [Figs. 4(a) and 4(d)], two pairs of 2D bands are evidently recognized: The upper pair of bands $S_{b}^{ \pm}$is degenerated at binding energy of $-10 \mathrm{meV}$ at $\bar{\Gamma}$, and disperses along the upper edge of bulk LH. At $\left|k_{\|}\right|>0.05-0.1 \AA^{-1}$, the bands disperse in projected bulk bands, suggesting these are surface resonances hybridized with bulk continuum. Note that the bands appear to be broken at $\sim 0.15 \AA^{-1}$, which is due to the finite slab geometry employed. The $S_{b}^{ \pm}$band pair is composed of two branches, $S_{b}^{+}$and $S_{b}^{-}$, which are spin polarized to the opposite directions, [1 112$]\left(S_{b}^{+}\right)$and [11 2$]\left(S_{b}^{-}\right)$ for $k_{\|}$in the [1 $\left.1 \overline{1} 0\right]$ direction. The spin polarization direction is reversed for $k_{\|}$in the [110] direction. These characteristics are in agreement with the Rashba spin splitting of a surface state band. 

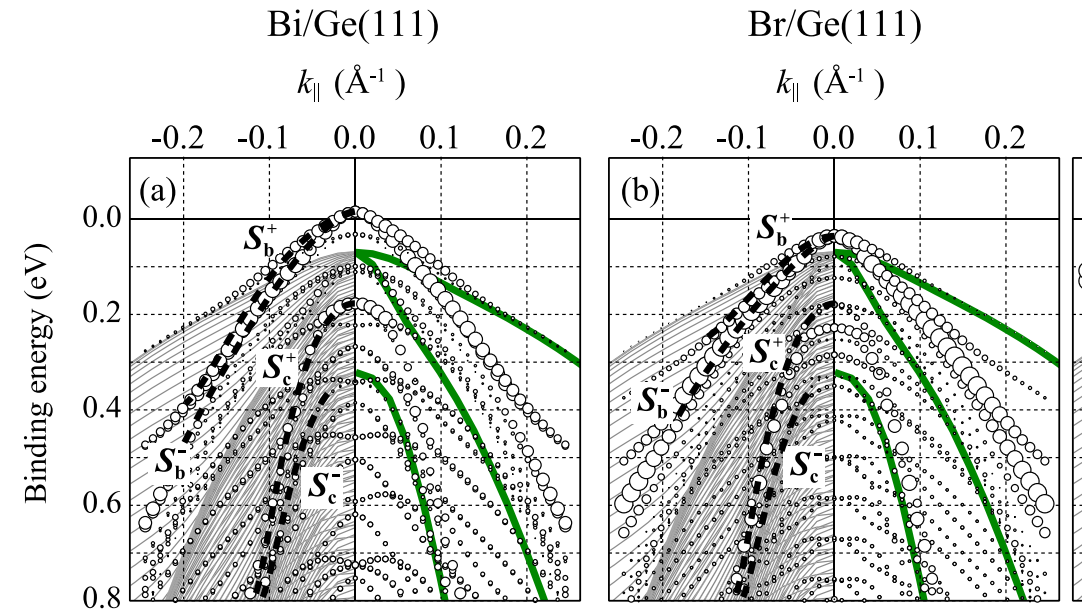

$$
\begin{gathered}
\mathrm{T} l / \mathrm{Ge}(111) \\
k_{\|}\left(\AA^{-1}\right)
\end{gathered}
$$
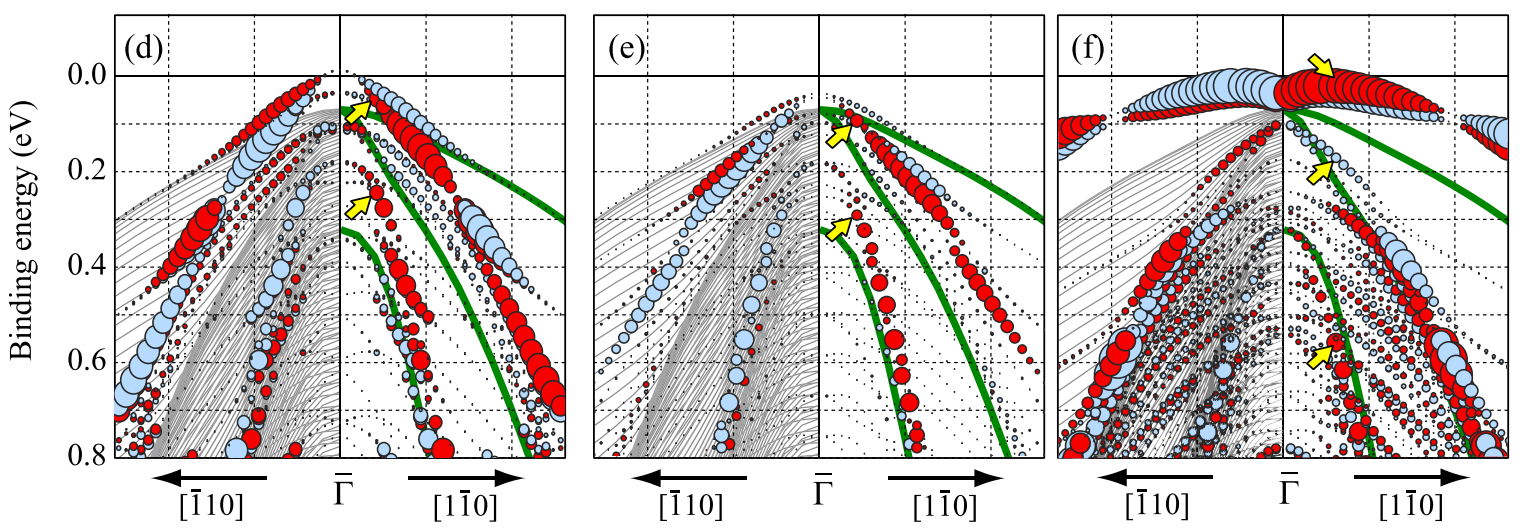

spin polarization toward $[11 \overline{2}] /[\overline{1} \overline{1} 2]$

FIG. 4. (Color online) Calculated band structures along [11̄0] for the (a, d) Bi/Ge, (b, e) Br/Ge, and (c, f) Tl/Ge slabs. The radii of the circles, $R_{k_{\|}, E}^{ \pm}$, are defined by Eq. (1). Panels (a-c) are depicted with $R_{k_{\|}, E}^{+}$and show total contribution of the subsurface layers. Panels (d-f) are depicted with $R_{k \|, E}^{-}$and show net spin polarization. The contrasts (colors, online) of the circles in (d-f) represent the spin polarization orientations. In the left side, the projected bulk valence bands (thin lines) obtained by the DFT calculation are shown. Solid lines in the right side represent the upper edges of the bulk $\mathrm{HH}, \mathrm{LH}$, and SO bands. The arrows in panels $(\mathrm{d}-\mathrm{f})$ indicate the states for which partial charge distribution is shown in Fig. 5. Panel (e) is reproduced from Ref. [7].

The other band pair, $S_{c}^{ \pm}$, exhibits a peculiar dispersion. The branches, $S_{c}^{+}$and $S_{c}^{-}$, disperse almost parallel to each other at $\left|k_{\|}\right|>0.05 \AA^{-1}$ along the upper edge of SO. Note that these branches are spin polarized toward the directions opposite to $S_{b}^{+}$and $S_{b}^{-}$. The branches do not degenerate with each other at $\bar{\Gamma}$. The $S_{c}^{+}$branch disperses into the bulk spin-orbit gap and is located at $\sim 100 \mathrm{meV}$ below VBM at $\bar{\Gamma}$. The $S_{c}^{-}$branch merges into SO upon approaching $\bar{\Gamma}$. The results for Br/Ge [Figs. 4(b) and $4(\mathrm{e})]$ are qualitatively very similar to the results for $\mathrm{Bi} / \mathrm{Ge}$ described above. The $S_{b}^{ \pm}$pair is located at a binding energy larger by $\sim 50 \mathrm{meV}$ than that of $\mathrm{Bi} / \mathrm{Ge}$.

On Tl/Ge, there also are spin-polarized bands $S_{b}^{ \pm}$and $S_{c}^{ \pm}$, dispersing along $\mathrm{LH}$ and SO, respectively, as shown in Figs. 4(c) and 4(f). They are located at higher binding energies than those on $\mathrm{Bi} / \mathrm{Ge}$ and $\mathrm{Br} / \mathrm{Ge}$. The $S_{c}^{+}$branch is positioned at $\sim 250 \mathrm{meV}$ below VBM as is evident in the spin-integrated plot [Fig. 4(c)]. In addition, there is another pair of spinpolarized surface bands, $S_{a}^{ \pm}$, which exhibits Rashba-type spin degeneracy at $\sim 30 \mathrm{meV}$ above $\mathrm{VBM}$ at $\bar{\Gamma}$ and disperse above $\mathrm{HH}$. The spin-split branches of $S_{a}^{ \pm}$cross each other at around $\left|k_{\|}\right|=0.2 \AA^{-1}$ and interchange the spin polarization orientation. This is ascribed to the $k_{\|}^{3}$ term of the Rashba Hamiltonian [24]. The existence of the $k_{\|}^{3}$ term is consistent with the fact that the $S_{a}^{ \pm}$bands change the spin polarization toward the out-of-plane direction as they approach $\bar{K}[14,25]$, since the $k_{\|}^{3}$ term is required to show the out-of-plane spin polarization [26]. Note that the out-of-plane spin components induced by the $k_{\|}^{3}$ term are not inverted due to the band crossing (see Fig. 2 in Ref. [14]).

The dispersion of the calculated bands $S_{a}$ and $S_{c}$ show a good overall agreement with that of $S_{1}$ and $S_{3}$ observed by ARPES, respectively. The $S_{b}$ band on Tl/Ge also agrees with the $S_{2}$ band observed by ARPES. The $S_{b}$ band on Bi/Ge and $\mathrm{Br} / \mathrm{Ge}$ are consistent with weak features observed by ARPES shown in Fig. 3. The spin-polarized peaks observed by spinresolved ARPES for $\mathrm{Bi} / \mathrm{Ge}$ and $\mathrm{Br} / \mathrm{Ge}$ are shown by triangles in Figs. 2(a) and 2(b) [6,7], which indicates a good agreement with the calculated spin texture of $S_{b}^{ \pm}$and $S_{c}^{+}$.

Figure 5 shows the layer-resolved partial charge distribution for the selected states belonging to the spin-polarized bands calculated for the $\mathrm{Bi} / \mathrm{Ge}, \mathrm{Br} / \mathrm{Ge}$, and $\mathrm{Tl} / \mathrm{Ge}$ slabs. The envelopes of the charge distribution for $S_{b}$ and $S_{c}$ exhibit 

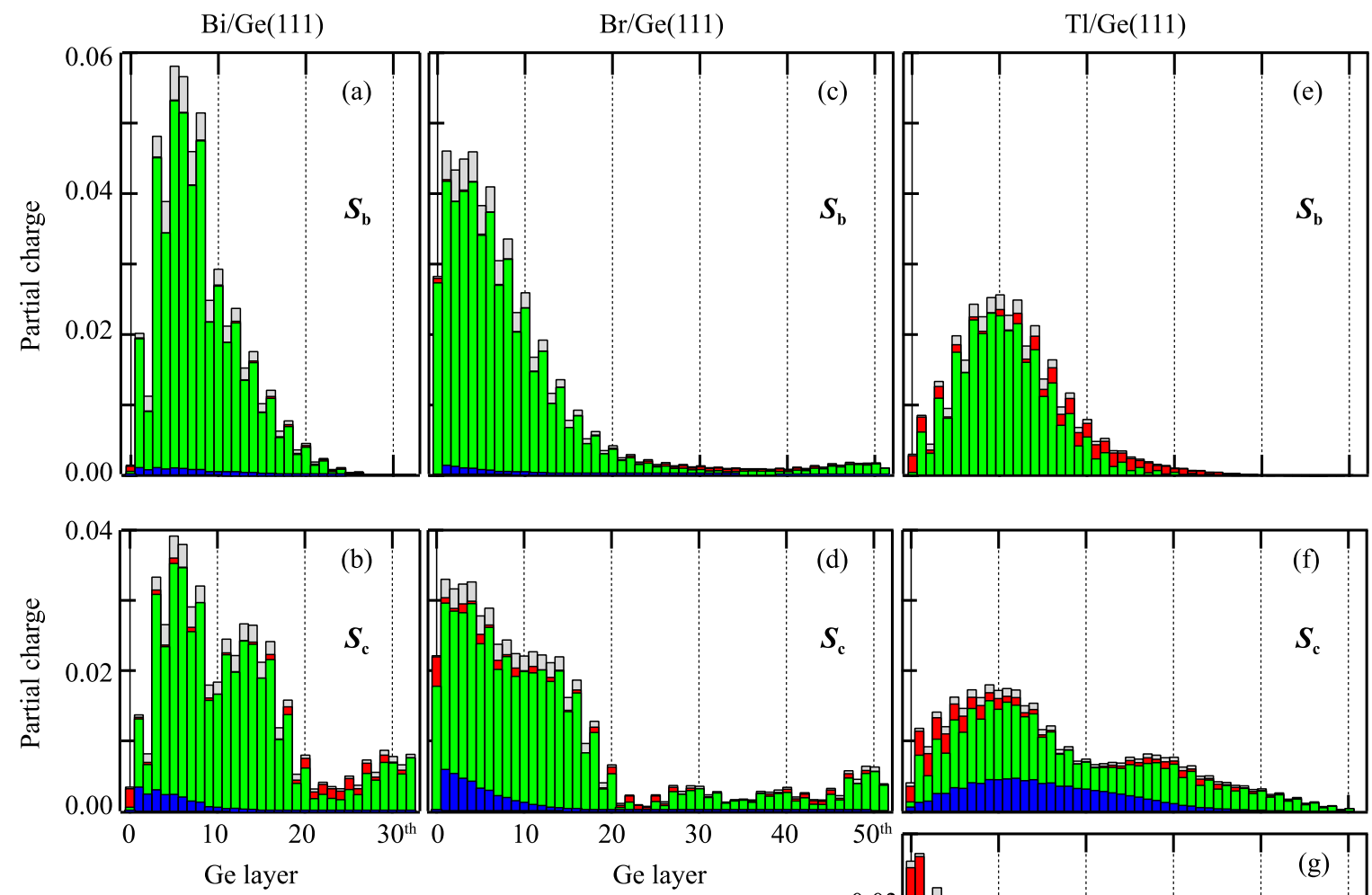

Ge layer

Ge layer
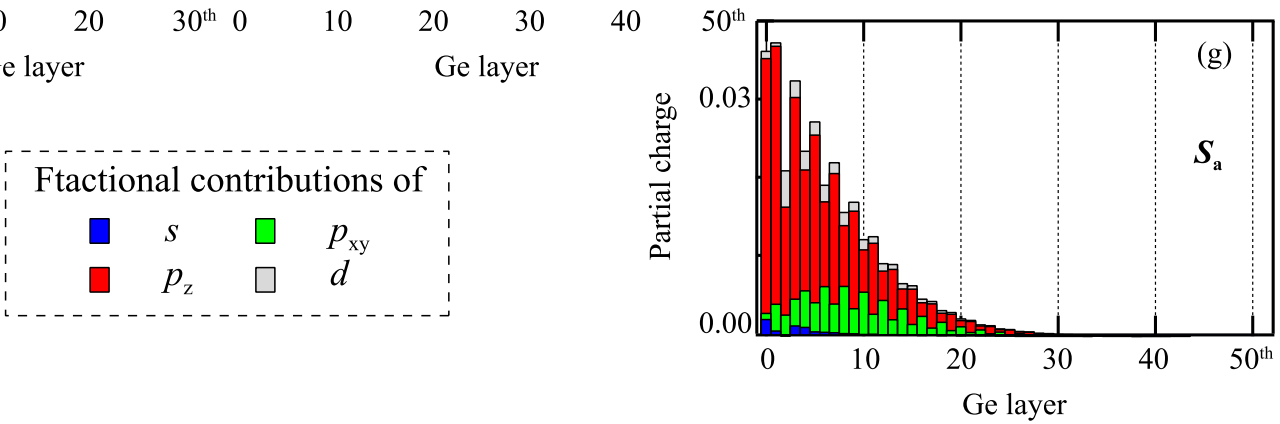

FIG. 5. (Color online) Layer- and spin-resolved partial charges of the states belonging to the spin-polarized surface bands on (a, b) Bi/Ge, (c, d) $\mathrm{Br} / \mathrm{Ge}$, and (e-g) $\mathrm{Tl} / \mathrm{Ge}$ at $k_{\|}$around $0.05 \AA^{-1}$ (indicated by arrows in Fig. 4). The zeroth layer corresponds to the adsorbate atoms.

broad maxima between the fifth and tenth Ge layers and then gradually decay with the depth, while that for $S_{a}$ exhibits monotonous decay. They mostly diminish at the other edge of the slab. These indicate that the spin-polarized states $S_{a}^{ \pm}, S_{b}^{ \pm}$, and $S_{c}^{ \pm}$are derived mainly from the atomic orbitals of Ge atoms in the subsurface layers. The contrasts (colors, online) of the bars represent the fraction of the contributions from $s, p_{x y}, p_{z}$, and $d$ orbitals. It is clearly seen that $S_{b}$ is derived almost solely from $p_{x y}$ orbitals. The $S_{c}$ band has also a dominant contribution from $p_{x y}$ orbitals but has a finite $(\sim 10 \%) s$ character. The $S_{a}$ band on $\mathrm{Tl} / \mathrm{Ge}$ shows, in clear contrast with the others, a dominant contribution from $p_{z}$ orbitals.

The spin polarization of each state was $80 \%$ for $S_{a}$ on Tl/Ge and $0.5-0.7$ for $S_{b}$ and $S_{c}$ for three surfaces, in agreement with the experimental value $\sim 0.5$ estimated for $S_{2}$ based on the spin-resolved ARPES data for $\mathrm{Bi} / \mathrm{Ge}$ [6]. Note that the partial charge distribution of $S_{c}$ exhibits the slowest decay into the bulk. The wave function is not perfectly damped at the opposite side of the slab, which is indicated by the bumps at the opposite sides of the slabs for $\mathrm{Bi} / \mathrm{Ge}$ and $\mathrm{Br} / \mathrm{Ge}$. However, the contribution of these bumps are at most $10 \%$ of the total wave functions. The variation of the slab thickness did not give any significant effect on the band dispersion or the spin polarization.

\section{DISCUSSION}

Surface states which originate from bulk bands, such as Shockley states on (111) surfaces of noble metals, localize in surface and subsurface layers. The partial charge distribution of these states show an exponential decay with the depth. In principle, this type of surface states can be formed individually from each bulk band (e.g., HH, LH, and SO of diamondtype semiconductors) and can be labeled with the irreducible representation of the corresponding bulk bands. The surface states disperse nearly parallel to the edges of the projected bulk bands as shown in Fig. 6(b). This picture well describes the subsurface states observed and calculated in this work. Surface states derived from the Ge bulk bands are also observed on other surfaces, such as $\mathrm{Pb} / \mathrm{Ge}(111)[9,10]$ and $\mathrm{Au} / \mathrm{Ge}(111)$ [11]. These surface bands can be interpreted as subsurface states derived from the bulk LH or SO bands similarly as those studied in this work. 
(a)

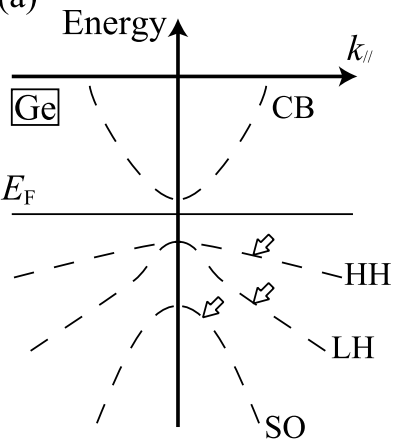

(c)

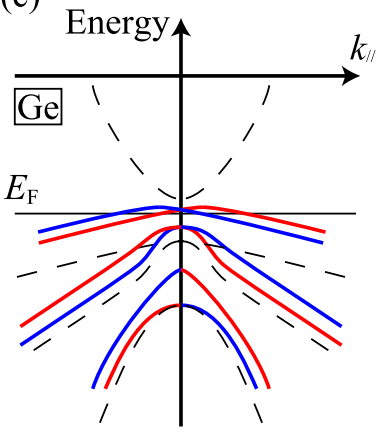

(b)

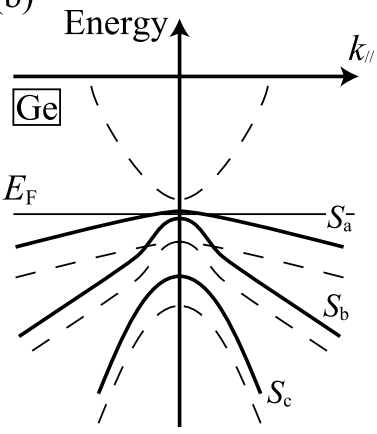

(d)

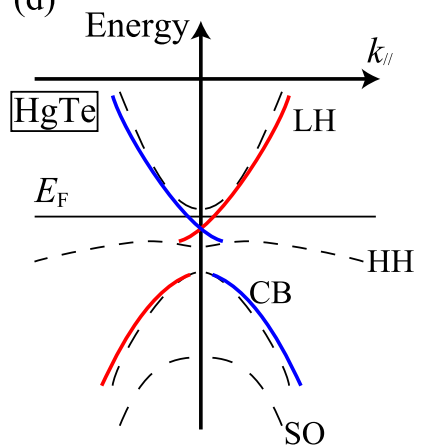

FIG. 6. (Color online) (a) Schematics for bulk band structures around $\Gamma$ for Ge. Arrows guide the positions of states listed in Table III. (b) The hypothetical surface bands derived from bulk SO and LH bands (solid lines). In this picture, the SOI for the surface bands is ignored for the sake of simplicity. (c) Subsurface-state bands with spin splitting due to SOI. The spin polarization directions are represented by the color of the lines. (d) Same as (c) for HgTe with uniaxial strain. Solid lines represent the topological gapless bands calculated on the surface [5].

In order to compare the orbital character of the subsurface states with those of the bulk states, we analyzed the fractional contributions of the atomic orbitals for bulk states around $\Gamma$. We chose three bulk states, each belonging to $\mathrm{HH}, \mathrm{LH}$, and SO bands as indicated in Fig. 6(a). The fractional orbital contributions of the states are summarized in Table I. For the LH state, $4 p_{x y}$ contributes twice as much as $4 p_{z}$, indicating that $p_{x}, p_{y}$, and $p_{z}$ orbitals contribute to a comparable extent. The HH state has a dominant $p_{z}$ contribution. The SO state has a predominant contribution from $p_{x}$ and $p_{y}$. Note also that the $4 s$ orbital makes the largest contribution to the SO state.

On the $\mathrm{Bi} / \mathrm{Ge}$ and $\mathrm{Br} / \mathrm{Ge}$ surfaces, the dangling bond, which is of $4 p_{z}$ character, is directly bonded with the $p_{z}$ orbitals of the adatoms on the $T_{1}$ sites. As a result, less-dispersive

TABLE I. The fractional contributions of atomic orbitals to the states belonging to the bulk states belonging to the $\mathrm{HH}, \mathrm{SO}$, and $\mathrm{LH}$ bands located near the subsurface states $\left(k_{\|}=0.05 \AA^{-1}\right)$.

\begin{tabular}{lcccc}
\hline \hline & $4 s$ & $4 p_{z}$ & $4 p_{x y}$ & $3 d$ \\
\hline HH & 0.00 & 0.47 & 0.41 & 0.11 \\
LH & 0.06 & 0.28 & 0.56 & 0.10 \\
SO & 0.10 & 0.13 & 0.68 & 0.09 \\
\hline \hline
\end{tabular}

bonding and antibonding states with $p_{z}$ characters are formed at $0.5-1.0 \mathrm{eV}$ below and above $E_{\mathrm{F}}$, respectively [27]. This results in the negligible $p_{z}$ character of the remaining $S_{b}$ and $S_{c}$ states on $\mathrm{Bi} / \mathrm{Ge}$ and $\mathrm{Br} / \mathrm{Ge}$. On the other hand, the $\mathrm{Tl}$ atoms occupy the $T_{4}$ sites on $\mathrm{Tl} / \mathrm{Ge}(111)-(1 \times 1)$, and hence the overlap between the Ge dangling bonds and the Tl orbitals are only moderate. As a result, the $S_{a}$ state is formed from Tl $6 p_{z}$ and $\mathrm{Ge} 4 p_{z}$ near VBM. The charge distribution [Fig. 5(g)] indicates comparable contribution from both $\mathrm{Tl}$ and topmost $\mathrm{Ge}$ atoms. The monotonous but gradual decay and increasing fraction of $p_{x y}$ contribution with the depth indicate that the $S_{a}$ surface state has a $\mathrm{HH}$-like character in the deeper layers. The contribution of Ge $4 p_{z}$ to the $S_{b}$ and $S_{c}$ bands are small also in this case. The difference between $S_{b}$ and $S_{c}$ is a larger contribution of Ge $4 s$ to $S_{c}$ as found in Fig. 5. This might be due to the formation mechanism that the $S_{b}$ and $S_{c}$ bands are originated mainly from LH and SO, respectively.

The subsurface states thus formed are Rashba spin polarized as shown in Fig. 6(c) due to the space-inversion asymmetry [8]. The size of spin splitting is several tens of $\mathrm{meV}$ which is consistent with the size of atomic SOI of Ge $4 p$ [28]. This is consistent with the fact that these states are localized in the subsurface layers and the spin splitting is governed by atomic SOI of the substrate Ge atoms.

The subsurface states on Ge have some similarities to the gapless surface states on TI: Both states are closely related to the bulk band structure but are formed near the surface with wave functions gradually decaying into the bulk. Since the gapless states in diamondlike lattices (e.g., HgTe with a zincblende lattice) disperse along the inverted bulk $\mathrm{LH}$ band above $E_{\mathrm{F}}$ as depicted in Fig. 6(d) [5], the LH-derived subsurface states we observed in this work would become gapless upon the inversion of the bulk bands if $\Delta_{S O}$ of Ge were larger and the bulk band gap were decreased. Actually, the subsurface states on HgTe were shown to change continuously to the gapless surface states upon the opening of the bulk band gap in HgTe due to the strain [5]. It was recently also shown that ultrathin films of $\alpha-\operatorname{Sn}(001)$, which is a TI with diamond lattice, have a gapless surface state dispersing parallel to both inverted bulk LH and conduction bands [29,30].

As shown by the DFT calculation, the $S_{c}^{+}$branch disperses across the bulk spin-orbit gap at $k_{\|}$close to $\bar{\Gamma}$ and reaches 50 and $100 \mathrm{meV}$ below VBM for $\mathrm{Bi} / \mathrm{Ge}$ and $\mathrm{Br} / \mathrm{Ge}$, respectively, while its partner $S_{c}^{-}$is merged into the bulk SO band, thus the spin degeneracy at $\bar{\Gamma}$ is lifted. This is unusual for the spin-split surface states due to the Rashba effect, which predicts the spin degeneracy at $\bar{\Gamma}$. The data of the spin-resolved ARPES study for $\mathrm{Bi} / \mathrm{Ge}[6]$ exhibit spin-polarized components at a binding energy $E_{B}=0.2 \mathrm{eV}$ and $k_{\|} \simeq \pm 0.03 \AA^{-1}$ are assigned to the $S_{c}^{+}$band. On the other hand, we could not identify, by spin-resolved ARPES, the $S_{c}^{-}$band which should be located at $0.4 \mathrm{eV}$ and counter-spin-polarized. The calculation (Fig. 4) indicates that the $S_{c}^{-}$band is merged into the bulk SO band and its spin polarization diminishes, which is in agreement with the experiment.

It should be pointed out that the $S_{c}^{ \pm}$branches are surface resonances, which means that each branch may change its partner to an arbitrary band that may be only weakly localized at the surface. The situation could be understood as an analogy with the gapless spin-split states on TI. On TI, SOI causes the 
inversion of an energetic order of bulk bands at high-symmetry points in the Brillouin zone. In a similar way, SOI in bulk Ge also inverts the energetic order of bulk light-hole and spin-orbit split-off bands around $\Gamma$. While it causes the finite gap in the infinite 3D Ge crystal, there might be formed a spin-polarized surface state, similarly to the spin-split states on TI, dispersing across the spin-orbit gap, which we suppose the origin of the peculiar dispersion of $S_{c}^{+}$. Note that the spin-polarized surface states on the Ge surfaces are not robust against structural perturbation, because bulk Ge has a trivial band structure.

\section{CONCLUSION}

In this work, we report on the details of the spinpolarized $\bar{\Gamma}$ surface electronic states on $\mathrm{Bi} / \mathrm{Ge}(111)-(\sqrt{3} \times$ $\sqrt{3}) R 30^{\circ}, \mathrm{Br} / \mathrm{Ge}(111)-(1 \times 1)$, and $\mathrm{Tl} / \mathrm{Ge}(111)-(1 \times 1)$. The first-principles calculations combined with ARPES results revealed the detailed nature of the spin-polarized 2D states localized in subsurface layers. They are closely related to the bulk HH, LH, and SO bands and are formed as surface resonances localized in subsurface layers due to the truncation of bulk crystals at the surface.
[1] M.-C. Desjonquères and D. Spanjaard, Concepts in Surface Physics, 2nd ed. (Springer-Verlag, Berlin, Heidelberg, 1996).

[2] H. Ibach, Physics of Surfaces and Interfaces (Springer-Verlag, Berlin, Heidelberg, 2006).

[3] W. Mönch, Semiconductor Surfaces and Interfaces, 3rd ed. (Springer, New York, 2001).

[4] M. Z. Hasan and C. L. Kane, Rev. Mod. Phys. 82, 3045 (2010).

[5] R.-L. Chu, W.-Y. Shan, J. Lu, and S.-Q. Shen, Phys. Rev. B 83, 075110 (2011).

[6] Y. Ohtsubo, S. Hatta, K. Yaji, H. Okuyama, K. Miyamoto, T. Okuda, A. Kimura, H. Namatame, M. Taniguchi, and T. Aruga, Phys. Rev. B 82, 201307(R) (2010).

[7] Y. Ohtsubo, S. Hatta, N. Kawai, A. Mori, Y. Takeichi, K. Yaji, H. Okuyama, and T. Aruga, Phys. Rev. B 86, 165325 (2012).

[8] E. I. Rashba, Sov. Phys. Solid State 2, 1109 (1960); Y. A. Bychkov and E. I. Rashba, JETP Lett. 39, 78 (1984).

[9] S.-J. Tang, T.-R. Chang, C.-C. Huang, C.-Y. Lee, C.-M. Cheng, K.-D. Tsuei, H.-T. Jeng, and C.-Y. Mou, Phys. Rev. B 81, 245406 (2010).

[10] K. Yaji, S. Hatta, T. Aruga, and H. Okuyama, Phys. Rev. B 86, 235317 (2012); K. Yaji, S. Hatta, Y. Ohtsubo, and T. Aruga (unpublished).

[11] K. Nakatsuji, R. Niikura, Y. Shibata, M. Yamada, T. Iimori, F. Komori, Y. Oda, and A. Ishii, Phys. Rev. B 84, 035436 (2011).

[12] S. Hatta, T. Aruga, Y. Ohtsubo, and H. Okuyama, Phys. Rev. B 80, 113309 (2009).

[13] Y. Ohtsubo, H. Muto, K. Yaji, S. Hatta, H. Okuyama, and T. Aruga, J. Phys.: Condens. Matter 23, 435001 (2011).

[14] Y. Ohtsubo, S. Hatta, H. Okuyama, and T. Aruga, J. Phys.: Condens. Matter 24, 092001 (2012).

[15] P. Blaha, K. Schwarz, G. Madsen, D. Kvasnicka, and J. Luitz, WIEN2k: An Augmented Plane Wave + Local Orbitals Program for Calculating Crystal Properties (Karlheinz Schwarz, Techn. Universität Wien, Wien, 2001).
[16] A. D. Becke and E. R. Johnson, J. Chem. Phys. 124, 221101 (2006).

[17] F. Tran and P. Blaha, Phys. Rev. Lett. 102, 226401 (2009).

[18] C. D. Thurmond, J. Electrochem. Soc. 122, 1133 (1975).

[19] S. Hatta, C. Kato, N. Tsuboi, S. Takahashi, H. Okuyama, T. Aruga, A. Harasawa, T. Okuda, and T. Kinoshita, Phys. Rev. B 76, 075427 (2007).

[20] Y. Ohtsubo, S. Hatta, M. Iwata, K. Yaji, H. Okuyama, and T. Aruga, J. Phys.: Condens. Matter 21, 405001 (2009).

[21] D. J. Chadi, Phys. Rev. B 16, 790 (1977).

[22] E. A. Kraut, R. W. Grant, J. R. Waldrop, and S. P. Kowalczyk, Phys. Rev. Lett. 44, 1620 (1980).

[23] M. Perego, G. Seguini, and M. Fanciulli, J. Appl. Phys. 100, 093718 (2006).

[24] L. Moreschini, A. Bendounan, I. Gierz, C. R. Ast, H. Mirhosseini, H. Höchst, K. Kern, J. Henk, A. Ernst, S. Ostanin, F. Reinert, and M. Grioni, Phys. Rev. B 79, 075424 (2009).

[25] K. Sakamoto, T. Oda, A. Kimura, K. Miyamoto, M. Tsujikawa, A. Imai, N. Ueno, H. Namatame, M. Taniguchi, P. E. J. Eriksson, and R. I. G. Uhrberg, Phys. Rev. Lett. 102, 096805 (2009).

[26] R. Winkler, Spin-Orbit Coupling Effects in Two-Dimensional Electron and Hole Systems (Springer-Verlag, Berlin, Heidelberg, 2003).

[27] K. Yaji, Y. Ohtsubo, S. Hatta, H. Okuyama, K. Miyamoto, T. Okuda, A. Kimura, H. Namatame, M. Taniguchi, and T. Aruga, Nat. Commun. 1, 17 (2009).

[28] S. Kotochigova, Z. H. Levine, E. L. Shirley, M. D. Stiles, and C. W. Clark, Phys. Rev. A 55, 191 (1997); http://physics.nist.gov/PhysRefData/DFTdata/ (1997).

[29] A. Barfuss, L. Dudy, M. R. Scholz, H. Roth, P. Höpfner, C. Blumenstein, G. Landolt, J. H. Dil, N. C. Plumb, M. Radovic, A. Bostwick, E. Rotenberg, A. Fleszar, G. Bihlmayer, D. Wortmann, G. Li, W. Hanke, R. Claessen, and J. Schäfer, Phys. Rev. Lett. 111, 157205 (2013).

[30] Y. Ohtsubo, P. Le Fèvre, F. Bertran, and A. Taleb-Ibrahimi, Phys. Rev. Lett. 111, 216401 (2013). 